\title{
Cationic surfactants for demulsification of produced water from alkaline-surfactant- polymer flooding
}

\begin{abstract}
In this research, demulsification of produced water (which is an oil-in-water emulsion) from alkaline-surfactant-polymer flooding, containing sodium alkyl sulfate, was evaluated using five different surfactants from the classes of nonionic, amphoteric, and cationic. It was observed that only single-tail cationic surfactants, namely, dodecyltriemthylammonium chloride (DTAC) and alkyltrimethylammonium bromide (ATAB), with a concentration of $1000 \mathrm{ppm}$, were capable of attaining transparent separated water phases following $3 \mathrm{~h}$ separation at room temperature with relative separation efficiencies, determined using fluorescence spectroscopy, of 89.4 and 59\%, respectively. However, the cationic surfactant dimethyldioctadecylammonium chloride (DDOAC) could achieve a relative separation efficiency of only $28.4 \%$ after 13 days, in contrast to nonionic and amphoteric surfactants that did not reveal any progress in demulsification. Similarly, given the demulsifier concentration of $1000 \mathrm{ppm}$, only DTAC and ATAB reduced the negative surface charge of oil droplets in the produced water after $3 \mathrm{~h}$ separation, and large droplets were formed owing to their coalescence after the addition of the respective demulsifiers as viewed by optical microscopy. The dominant emulsification mechanism is believed to be electrostatic stabilization. Consequently, the proposed demulsification mechanism is the formation/adsorption of cationic-anionic pairs at the oil-water interface. When comparing the demulsification performances of various demulsifying surfactants, although high interfacial activity (low interfacial tension (IFT)) is an essential feature for a demulsifier to be considered effective, it was concluded that lower equilibrium IFT does not necessarily result in superior separation efficiency, and other parameters such as type, tail branch number (i.e., single-tail or double-tail), and purity of surfactant may have profound effects on both separation efficiency and demulsification speed of the emulsion. From the dynamic IFT data, it was realized that DTAC and ATAB caused faster demulsification than DDOAC. The undesirable demulsification performance of DDOAC might have been due to its double-tail structure, which confined its interfacial adsorption. The operational variables, including salinity, $\mathrm{pH}$, and temperature, in the demulsification process by DTAC were optimized with respect to the changes of IFT, and the optimum values were found to be 2 wt $\%, 7.0$, and $35^{\circ} \mathrm{C}$, respectively.
\end{abstract}

Keyword: Emulsions; Interfaces; Lipids; Liquids; Surfactants 